\title{
Diferir la implantación de stents coronarios en la angioplastia primaria: una visión crítica basada en la evidencia disponible
}

\author{
Deferring stenting in primary angioplasty: a critical view based on available evidence \\ Diego Fernández-Rodríguez*, Kristian Rivera y Juan Casanova \\ Servicio de Cardiología, Hospital Universitari Arnau de Vilanova, Institut de Recerca Biomèdica de Lleida, Lleida, España
}

Encontramos muy interesante el trabajo de Custodio-Sánchez, et al. ${ }^{1}$ sobre la utilidad de diferir el implante de stents en pacientes remitidos para angioplastia primaria (AP) en los que persiste una alta carga de trombo residual tras preparar la lesión culpable.

Se evaluaron un total de 833 pacientes remitidos para AP. Los pacientes fueron reclasificados en función de la carga de trombo residual tras pasar la guía de angioplastia y realizar predilatación con balón y/o tromboaspiración, en los que se obtenía un flujo Thrombolysis In Myocardial Infarction Flow (TIMI) 2-3 sobre la arteria responsable del infarto. De 47 pacientes con alta carga de trombo residual, 19 recibieron implante inmediato de stent y en 28 pacientes se retrasó el implante, según el criterio del operador. Se observó que el grupo de implante diferido presentaba mejores indicadores de perfusión miocárdica, evaluados mediante la escala Thrombolysis In Myocardial Infarction Myocardial Perfusion (TMP), y se evitó el implante de stents en un $56 \%$ de los pacientes, aunque durante el seguimiento no se detectaron diferencias significativas entre los grupos en cuanto al pronóstico clínico. No se realizaron estudios adicionales más específicos, como la resonancia magnética cardíaca, para una mejor evaluación del estado de la microcirculación.

Los beneficios de una estrategia de implante diferido de stents consistiría hipotéticamente en preservar la integridad de la microvasculatura, al evitar la embolización y el no reflujo asociado con el implante inmediato de stents en la AP. Asimismo, el ahorro de stents en determinados pacientes podría evitar la aparición de complicaciones, como reestenosis o trombosis de stents. Sin embargo, la estrategia diferida ha presentado resultados contradictorios sobre la preservación microvascular en estudios de resonancia ${ }^{2,3}$, y el principal estudio que evalúa eventos clínicos (DANAMI-3-DE$\mathrm{FER})^{4}$, así como un metaanálisis de ensayos clínicos ${ }^{5}$, no objetivaron beneficios en las variables de valoración clínica duras, asociándose, incluso, a mayor necesidad de revascularización del vaso diana ${ }^{4}$. Estos hechos, unidos a la inherente necesidad de recateterización en esta estrategia, ha llevado a que las sociedades científicas no avalen su uso rutinario ${ }^{6}$.

Basándonos en lo previo aportamos ciertas consideraciones:

- Tamaño muestral: El reducido tamaño del estudio, debido a su carácter unicéntrico y a la dificultad de reclutar este tipo de pacientes, como confirman estudios similares ${ }^{6,7}$, no permitió cuantificar como significativas múltiples diferencias en características clínicas y angiográficas, así como en los resultados clínicos, lo que pudo afectar a las conclusiones del estudio.

- Reducción de la carga trombótica: La implantación de stents en la fase aguda del infarto se asocia con

\section{Correspondencia:}

*Diego Fernández-Rodríguez

Hospital Universitari Arnau de Vilanova

Av. Alcalde Rovira Roure, 80

Disponible en internet: 08-05-2019

C.P. 25198, Lleida, España

Fecha de aceptación: 09-03-2019

Arch Cardiol Mex. 2019;89(2):201-202

DOI: 10.24875/ACM. 19000095

www.archivoscardiologia.com

1405-9940 @ 2019 Instituto Nacional de Cardiología Ignacio Chávez. Publicado por Permanyer México SA de CV. Este es un artículo Open Access bajo la licencia CC BY-NC-ND (http://creativecommons.org/licenses/by-nc-nd/4.0/). 
embolización trombótica. Actualmente, la principal estrategia para reducir la carga trombótica es la trombectomía manual, y a pesar de no recomendarse rutinariamente, es una herramienta muy útil en pacientes con alta carga trombótica ${ }^{6,8}$. En el presente trabajo, más de un $40 \%$ de pacientes por grupo no recibió trombectomía. Esto pudo haber incrementado la embolización y empeorado la perfusión miocárdica, especialmente tras el implante inmediato del stent.

- Ectasia coronaria: Los autores hipotetizan que la estrategia diferida podría asociarse con implante de stents de mayor diámetro en el segundo procedimiento, al reducirse la vasoconstricción aguda. Sin embargo, en sus datos no se observaron diferencias significativas en el diámetro de los stents finalmente implantados $(4 \pm 0.7 \mathrm{~mm}$ vs. $3.7 \pm 0.7 \mathrm{~mm} ; p=N S)$, incluso cuando la estrategia diferida se asociaba con mayor prevalencia de ectasias coronarias ( $57 \%$ vs. $16 \% ; p=0.005)$. No obstante, concordamos que en casos extremos de ectasia coronaria, la ausencia de disponibilidad de stents de diámetro suficiente para lograr una adecuada aposición podría ser una limitación al implante de stents en la fase aguda ${ }^{9}$.

- Prevención de la restenosis y trombosis de stent: El trabajo informa de una reducción superior al $50 \%$ en el implante de stents en el grupo de estrategia diferida. Sin embargo, este ahorro no se tradujo en beneficios clínicos, ya que el grupo de estrategia diferida presentó mayor porcentaje de revascularización del vaso diana, trombosis de stent, infarto recurrente y fallecimiento, aunque sin alcanzar la significación estadística, probablemente debido al reducido tamaño muestral. A día de hoy, la baja tasa de reestenosis y trombosis con el uso de stents farmacoactivos de nueva generación en el infarto ${ }^{10}$ ha reducido el interés por la estrategia diferida y las guías no recomiendan su uso extensivo ${ }^{6}$.
Una aportación relevante del presente estudio ${ }^{1}$ es describir que una estrategia de implante diferido de stents, restringida a pacientes con alta carga trombótica residual, podría ser una alternativa a la estrategia convencional en la AP. Este trabajo refuerza la necesidad de ampliar la investigación acerca del papel de la carga trombótica residual sobre la función microvascular y el pronóstico a largo plazo en estos pacientes.

\section{Bibliografía}

1. Custodio-Sánchez $P$, Damas-De Los Santos F, Peña-Duque MA, Coutiño-Castelán D, Arias-Sánchez E, Abundes-Velasco A, et al. Deferred versus immediate stenting in patients with ST - segment elevation myocardial infarction and residual large thrombus burden reclassified in the culprit lesion. Arch Cardiol Mex. 2018; pii: S1405-9940(18)30032-6.

2. Casse SE, Belle L, Ndrepepa G, Bosson JL, Fusaro, Lønborg, et al. Deferred vs Immediate Stenting in Primary Percutaneous Coronary Intervention: A Collaborative Meta-analysis of Randomized Trials With Cardiac Magnetic Resonance Imaging Data. Can J Cardiol. 2018;34:1573-80.

3. Carrick D, Oldroyd KG, McEntegart M, Haig C, Petrie MC, Eteiba H, et al. $A$ randomized trial of deferred stenting versus immediate stenting to prevent no- or slow-reflow in acute ST-segment elevation myocardial nfarction (DEFER-STEMI). J Am Coll Cardiol. 2014;63:2088-98.

4. Kelbaek H, Hofsten DE, Kober L, Helqvist S, Klovgaard L, Holmvang L, et al. Deferred versus conventional stent implantation in patients with ST-segment elevation myocardial infarction (DANAMI 3-DEFER): an open-label, randomized controlled trial. Lancet. 2016;387:2199-206.

5. Mahmoud AN, Saad M, Elgendy AY, Mentias A, Elgendy IY. et al. Deferred or immediate stent implantation for primary percutaneous coronary intervention: Ameta-analysis of randomized trials. Catheter Cardiovasc Interv. 2018;91:260-4.

6. Ibanez B, James S, Agewall S, Antunes MJ, Bucciarelli-Ducci C, Bueno H, et al. 2017 ESC Guidelines for the management of acute myocardial infarction in patients presenting with ST-segment elevation. Eur Heart J. 2018;39:119-77.

7. Tang $\mathrm{L}$, Zhou $\mathrm{SH}, \mathrm{Hu} X \mathrm{XQ}$, Fang ZF, Shen XQ. Effect of delayed vs. immediate stent implantation on myocardial perfusion and cardiac function in patients with ST-segment elevation myocardial infarction undergoing primary percutaneous intervention with thrombus aspiration. Can J Cardiol. 2011;27:541-7.

8. Ahn SG, Choi HH, Lee JH, Lee JW, Youn YJ, Yoo SY, et al. The impact of initial and residual thrombus burden on the no-reflow phenomenon in patients with ST-segment elevation myocardial infarction. Coron Artery Dis. 2015;26:245-53.

9. Schram HCF, Hemradj VV, Hermanides RS, Kedhi E, Ottervanger JP; Zwolle Myocardial Infarction Study Group. Coronary artery ectasia, an independent predictor of no-reflow after primary $\mathrm{PCl}$ for ST-elevation myocardial infarction. Int J Cardiol. 2018;265:12-7.

10. Sabaté M, Brugaletta $S$, Cequier A, Iñiguez A, Serra A, Jiménez-Quevedo $\mathrm{P}$, et al. Clinical outcomes in patients with ST-segment elevation myocardial infarction treated with everolimus-eluting stents versus bare-metal stents (EXAMINATION): 5-year results of a randomised trial. Lancet. 2016:387:357-66. 